\title{
The Schizophrenia Risk Allele C of the TCF4 rs9960767 Polymorphism Disrupts Sensorimotor Gating in Schizophrenia Spectrum and Healthy Volunteers
}

\author{
Boris B. Quednow, ${ }^{1,2,9}$ Ulrich Ettinger, ${ }^{3,4}$ Rainald Mössner, ${ }^{2}$ Dan Rujescu, ${ }^{5}$ Ina Giegling, ${ }^{5}$ David A. Collier, ${ }^{6}$ \\ Anne Schmechtig, ${ }^{7}$ Kai-Uwe Kühn, ${ }^{2}$ Hans-Jürgen Möller, ${ }^{3}$ Wolfgang Maier, ${ }^{2}$ Michael Wagner, ${ }^{2 *}$ and Veena Kumari ${ }^{7,8 *}$ \\ ${ }^{1}$ University Hospital of Psychiatry, Division of Experimental and Clinical Pharmacopsychology, University of Zurich, CH-8032 Zurich, Switzerland, \\ ${ }^{2}$ Department of Psychiatry and Psychotherapy, University of Bonn, D-53105 Bonn, Germany, Departments of ${ }^{3}$ Psychiatry and ${ }^{4}$ Psychology and ${ }^{5}$ Division of \\ Molecular and Clinical Neurobiology, Ludwig-Maximilians University Munich, D-80337 Munich, Germany, ${ }^{6}$ Social, Genetic and Developmental Psychiatry \\ Centre, ${ }^{7}$ Department of Psychology, Institute of Psychiatry, King's College London, and ${ }^{8}$ National Institute for Health Research Biomedical Research Centre \\ for Mental Health, South London and Maudsley National Health Service Foundation Trust, SE5 8AF London, United Kingdom, and ${ }^{2}$ Zurich Center for \\ Integrative Human Physiology, University of Zurich, CH-8057 Zurich, Switzerland
}

In a large-scale meta-analysis, it has been recently shown that the transcription factor 4 (TCF4) gene is among the most prominent susceptibility genes for schizophrenia. Moreover, transgenic mice overexpressing TCF4 in the brain display a reduction of sensorimotor gating measured by prepulse inhibition (PPI) of the acoustic startle response (ASR). PPI is heritable and has been established as an important translational endophenotype of schizophrenia. We therefore investigated the impact of the schizophrenia susceptibility gene TCF4 (rs9960767) on sensorimotor gating of the ASR in healthy humans and in patients with a schizophrenia spectrum disorder. We assessed PPI, startle reactivity, and habituation of the ASR in two independent samples. The first sample consisted of 107 healthy volunteers from London, UK. The second sample was a schizophrenia spectrum group $(n=113)$ of 73 schizophrenia patients and 40 individuals at high risk for schizophrenia from Bonn, Germany (total sample $n=220$ ). In both samples, PPI was strongly decreased in carriers of the schizophrenia risk allele $\mathrm{C}$ of the TCF4 gene (meta-analysis across both samples: $p=0.00002$ ), whereas startle reactivity and habituation were unaffected by TCF4 genotype. Sensorimotor gating is modulated by TCF4 genotype, indicating an influential role of TCF4 gene variations in the development of early information-processing deficits in schizophrenia.

\section{Introduction}

Although schizophrenia is a strongly inherited disease with a heritability of $80 \%$ or more, the genetic basis of this disease is still elusive. A recent meta-analysis across SNP data from several large

Received Jan. 31, 2011; revised March 10, 2011; accepted March 20, 2011.

Author contributions: B.B.Q., U.E., R.M., D.A.C., K.-U.K., H.-J.M., W.M., M.W., and V.K. designed research; B.B.Q., U.E., R.M., D.R., I.G., D.A.C., A.S., K.-U.K., M.W., and V.K. performed research; D.R., I.G., and D.A.C. contributed unpublished reagents/analytic tools; B.B.Q., R.M., D.R., M.W., and V.K. analyzed data; B.B.Q., U.E., R.M., D.A.C., M.W., and V.K. wrote the paper.

Dr. Quednow was supported by the Swiss National Science Foundation (Grant PP00P1_123516). He also had full access to all of the data in the study and takes responsibility for the integrity of the data and the accuracy of the data analysis. Dr. Ettingerwas supported by the German Research Foundation (DFG) (GrantET 31/2-1). Dr. Wagnerwas supported by the DFG (Grant WA 737/7). Drs. Maier and Mössner were supported by the European Union (Grant FP7-Health-F4-2009-242257ADAMS) and by the German Federal Ministry for Education and Research (BMBF) (Grants POSITIVE 01GV0907, NGFN+ MooDs PNM-01GS08146-3). Dr. Collier, Dr. Ettinger, and Anne Schmechtig were supported by the European Commission (Grant FP6-2005-LIFESCIHEALTH-7, STREP project 037,761, SGENE). Dr. Kumari was supported by the Wellcome Trust (067427/z/02). The study was funded in part by the Federal Ministry of Education and Research within the scope of the German Research Network on Schizophrenia (Grants 01-GI-0232, 01-GI-0236, and 01-GI-9934). We are grateful to Tobias Bast (Centre for Cognitive and Neural Systems, University of Edinburgh), Anita Rauch (Institute of Medical Genetics, University of Zurich), and Lea Hulka (University Hospital of Psychiatry, University of Zurich) for helpful comments and suggestions to the manuscript. We thank Vera Guttenthaler for excellent technical assistance.

${ }^{*}$ M.W. and V.K. contributed equally to this work.

Correspondence should be addressed to Dr. Boris B. Quednow, Division of Experimental and Clinical Pharmacopsychology, University Hospital of Psychiatry, Lenggstrasse 31, CH-8032Zurich, Switzerland. E-mail: quednow@bli.uzh.ch.

DOI:10.1523/JNEUROSCI.0526-11.2011

Copyright $\odot 2011$ the authors $\quad 0270-6474 / 11 / 316684-08 \$ 15.00 / 0$ genomewide scans revealed that the risk of schizophrenia was significantly associated with a marker in intron four of the transcription factor 4 (TCF4) gene on chromosome 18q21.2 (Stefansson et al., 2009). Interestingly, a translational animal study recently showed that transgenic mice that moderately overexpress TCF4 postnatally in the brain display profound reductions in sensorimotor gating (Brzózka et al., 2010)—an established translational and behavioral endophenotype of schizophrenia (Gottesman and Gould, 2003). TCF4 is a class I basic helix-loophelix $(b H L H)$ protein that is involved in the development of the mammalian CNS. Although TCF4 shows sustained expression in several brain regions throughout lifetime, its function in the adult CNS remains widely unclear (Soosaar et al., 1994; Flora et al., 2007).

Sensorimotor gating is commonly operationalized by prepulse inhibition (PPI) of the acoustic startle response (ASR). PPI is defined as a substantial reduction of the startle amplitude that occurs when a distinctive nonstartling stimulus is presented 30 $500 \mathrm{~ms}$ before the startling stimulus (Graham, 1975). PPI is regulated by a cortico-striato-pallido-pontine (CSPP) circuitry including frontal and mediotemporal regions, ventral striatum, ventral pallidum, and pontine regions of the brainstem (Fendt et al., 2001; Swerdlow et al., 2001). Several neuropsychiatric disorders present diminished PPI (Braff et al., 2001), but this is most 
strongly evident in schizophrenia spectrum disorders (Braff et al., 1992; Cadenhead et al., 1993; Kumari et al., 2000; Parwani et al., 2000; Ludewig et al., 2003). Furthermore, PPI is heritable (Anokhin et al., 2003; Greenwood et al., 2007), reduced in unaffected relatives of schizophrenia patients (Cadenhead et al., 2000; Kumari et al., 2005a), influenced by SNPs within the dopamine, acetylcholine, and serotonin system (Quednow et al., 2008b, 2009, 2010; Roussos et al., 2008a,b; Petrovsky et al., 2010) and already decreased during the prodromal stage of schizophrenia (Quednow et al., 2008a), suggesting that PPI is an important and valid candidate as an intermediate or endophenotypic marker in genetic studies of schizophrenia (Gottesman and Gould, 2003).

Based on the recent finding that the TCF4 SNP rs9960767 is a genetic risk factor for schizophrenia, that transgenic mice overexpressing TCF4 show reduced sensorimotor gating, and that sensorimotor gating is an established endophenotype of schizophrenia, we hypothesized that human carriers of the schizophrenia risk allele C of the TCF4 rs9960767 SNP would exhibit decreased sensorimotor gating. To avoid spurious results, we investigated two independent European Caucasian samples-a sample of 107 healthy volunteers recruited in United Kingdom, and a combined sample of 73 schizophrenia inpatients and 40 individuals at high risk for schizophrenia recruited in Germany, to directly test for replicability of our findings. This allowed us to explore the impact of this SNP in a sample of healthy subjects with a normal expression of the PPI endophenotype as well as in a schizophrenia spectrum sample with a known alteration of the endophenotype.

\section{Materials and Methods}

Participants. For the first sample, 107 healthy Caucasian volunteers (54 males, 53 females) with self-reported grandparents of European origin were recruited through local advertisements in South London, United Kingdom. Participants aged between 18 and 45 years were screened for the exclusion criteria of DSM-IV Axis I disorders using the Structured Clinical Interview for DSM-IV Disorders for healthy subjects (SCIDNP). Additional exclusion criteria were a history of head injuries, any known neurological abnormalities or systemic illness with known neurological complication, a first-degree relative with psychosis or obsessive-compulsive disorder, and a history of substance abuse or dependence. Current smoking behavior was assessed with the Fagerström Test for Nicotine Dependence (Heatherton et al., 1991) and the number of cigarettes smoked per day (CPD).

For the second sample, 73 inpatients (50 males, 23 females) admitted to the psychiatric hospital of the University of Bonn, Germany, with a diagnosis of schizophrenia according to DSM-IV were included. Moreover, 40 subjects with symptoms indicating high risk for schizophrenia (23 males, 17 females) were recruited as described in detail previously (Häfner et al., 2004; Quednow et al., 2008a; Frommann et al., 2010). Patients and high-risk individuals were included if they were of Caucasian ethnicity with self-reported grandparents of European origin and aged between 18 and 65 years. Exclusion criteria were a history of head injuries, a neurological disease, a history of substance abuse or dependency, or a severe somatic disease. Every participant was evaluated with the Structured Clinical Interview (SCID-I). Clinical symptoms were measured with the Positive and Negative Syndrome Scale (PANSS) (Kay et al., 1992). Fourteen schizophrenia patients were never medicated (these patients experienced a first episode of psychosis), 12 patients received a typical antipsychotic, 43 patients were treated with an atypical antipsychotic, 3 received two atypical drugs, and 1 received a typical and an atypical antipsychotic. Four high-risk individuals received treatment with atypical antipsychotics. From schizophrenia patients, urine toxicologies were obtained before inclusion to rule out active substance abuse. The total (healthy and schizophrenia spectrum) sample consists of 220 individuals. For both samples, ethical approvals of the local ethics committees were obtained, and all participants provided written informed consent before inclusion.
Genotyping. In the healthy volunteers from London, DNA concentration from cheek swabs or from EDTA acid anticoagulated blood was adjusted using the PicoGreen quantitation reagent (Invitrogen) and $1 \mathrm{ng}$ was genotyped using the iPLEX assay on the MassARRAY MALDI-TOF mass spectrometer (SEQUENOM). Genotyping was successful in $93.5 \%$ of subjects. To check for genotyping errors, CEU families were genotyped too with a $100 \%$ concordance rate. Furthermore, genotype frequency of the TCF4 rs9960767 SNP in the London sample (AA: $87.8 \%$, AC: $10.2 \%$, CC: $2 \%$; A-allele: $92.9 \%$, C-allele: $7.1 \%$ ) was very similar as in the HapMap-CEU population available at the NCBI website (AA: $88.3 \%$, AC: $10.0 \%$, CC: $1.7 \%$; A-allele: $93.3 . \%$, C-allele: $6.7 \%$; www.hapmap. org) and distributed in accordance with the Hardy-Weinberg Equilibrium (HWE) $(p=0.07)$.

In the German schizophrenia patients and high-risk individuals, DNA for genotyping was isolated either from EDTA acid anticoagulated blood or permanent cell cultures received after transforming the lymphocytes with Epstein-Barr virus. The isolation of the DNA followed the QIAGEN protocol for the Blood \& Cell Culture DNA Maxi Kit (QIAGEN). PCR was performed using $12.5 \mathrm{ng}$ of DNA. The TCF4 SNP (rs9960767) was analyzed by a TaqMan assay (Applied Biosystems). Genotyping was successful in $97.3 \%$ of subjects. For quality control, the complete sample was genotyped twice. Concordance among duplicate samples was $100 \%$. Genotype frequency of the TCF4 SNP in the Bonn sample (AA: $89.5 \%$, AC: 10.5\%, CC: $0 \%$; A-allele: $94.8 \%$, C-allele: $5.2 \%$ ) was also comparable to the HapMap-CEU population (see above) and as well distributed in accordance with HWE $(p=1.0)$.

Startle response measurement. The two samples were assessed with the same hardware but with slightly different PPI paradigms. In the London sample, we used three stimulus onset asynchronies (SOAs) of 30, 60, and $120 \mathrm{~ms}$ between the prepulse and the pulse alone (PA), while we used only a single SOA condition in the Bonn sample (120 ms). Equipment, setup, PPI testing, and data acquisition and scoring procedures for both methods have previously been described in detail [London sample: $\mathrm{Ku}-$ mari et al. (2005b); Bonn sample: Quednow et al. (2006a,b, 2008a)]. In both centers, we used the SRRED2 software (San Diego Instruments) to analyze the startle data. According to Braff et al. (1992), the latency to startle response onset was defined by a shift of six digital units from the baseline value occurring between 21 and $120 \mathrm{~ms}$ after the acoustic startle stimulus. Latency to response peak was defined as the point of maximal amplitude that occurred within $150 \mathrm{~ms}$ after the startle stimulus. Response rejections were made in case of onset-to-peak latencies $>95 \mathrm{~ms}$, onset latencies $>100 \mathrm{~ms}$, and baseline shifts $>90$ digital units (e.g., due to spontaneous or voluntary blinks). Subjects with response rejections $>50 \%$ were excluded from data analysis (healthy subjects: $n=2$; schizophrenia patients: $n=3$; high-risk individuals: $n=2$ ). All remaining subjects showed a mean startle amplitude $>25$ units-the commonly used threshold for analyzable startle data (Braff et al., 1992).

The mean percentage PPI of startle amplitude was calculated using the following formula: $\%$ PPI $=100 \times($ amplitude on PA trials - amplitude on prepulse trials)/amplitude on PA trials (Braff et al., 1992). For the assessment of startle habituation, PA trials were each divided into four blocks in the British sample and in six blocks in the German sample. The calculation of the habituation measures (early and late habituation in percentage, and linear gradient coefficient $b$ ) has been described in detail previously (Quednow et al., 2006a,b). Startle reactivity was assessed by the mean amplitude of the first block of PA trials and the mean amplitude of all PA trials.

Statistical analysis. Given that the minor allele frequency of the TCF4 rs9960767 SNP is relatively low $(\sim 6 \%)$ (Stefansson et al., 2009), we compared only homozygous carriers of the A-allele with carriers of the schizophrenia risk allele C (AA vs AC + CC). All demographic data were analyzed by ANOVA with the exception of frequency data. Frequency data were analyzed using Fishers exact $\chi^{2}$ test. Given that sex, smoking, and antipsychotic medication might affect PPI (Swerdlow et al., 1997; Kumari and Gray, 1999; Swerdlow et al., 2006), these variables were introduced as covariates in analyses of covariance (ANCOVA) of PPI data independent of the statistical significance of the covariates. All other psychophysiological parameters were analyzed with ANOVA. The confirmatory statistical comparisons were performed at a significance level 
Table 1. Demographic data and psychophysiological parameters of healthy volunteers from London, UK, grouped according to their TCF4 rs9960767 genotype (means and SEMs in parentheses, sex and smoking status in frequency data)

\begin{tabular}{|c|c|c|c|c|c|c|c|c|}
\hline TCF4 rs9960767 genotype & $C C+A C$ & $\mathrm{AA}$ & Total & $F$ & $\mathrm{df} / \mathrm{df}_{\mathrm{err}}$ & $p$ & $d^{a}$ & $\eta_{p^{2}}$ \\
\hline$n$ & $12(12.2 \%)$ & $86(87.8 \%)$ & $98(100 \%)$ & & & & & \\
\hline Age & $25.4(1.7)$ & $26.2(0.7)$ & $26.1(0.6)$ & 0.20 & $1 / 97$ & 0.66 & 0.13 & 0.00 \\
\hline Years of education & $17.2(1.2)$ & $17.2(0.3)$ & $17.2(0.3)$ & 0.00 & $1 / 97$ & 1.00 & 0.00 & 0.00 \\
\hline Male $(\%)^{b}$ & $33.3 \%$ & $51.2 \%$ & $49.0 \%$ & - & 1 & 0.36 & - & - \\
\hline Current smokers (\%) ${ }^{b}$ & $0.0 \%$ & $26.7 \%$ & $23.5 \%$ & - & 1 & 0.06 & - & - \\
\hline $\begin{array}{l}\text { Current cigarettes smoked per day } \\
\text { (only in smokers) }\end{array}$ & - & $9.0(1.3), n=23$ & $9.0(1.3), n=23$ & - & - & - & - & - \\
\hline $\begin{array}{l}\text { Fagerström Nicotine Dependence } \\
\text { Test (score; only in smokers) }\end{array}$ & - & $1.8(0.5), n=23$ & $1.8(0.5), n=23$ & - & - & - & - & - \\
\hline $\begin{array}{l}\text { First block, amplitude of pulse-alone } \\
\text { trials (arbitrary units) }\end{array}$ & $685(160)$ & $706(41.9)$ & $703(41.3)$ & 0.03 & $1 / 97$ & 0.87 & 0.05 & 0.00 \\
\hline $\begin{array}{l}\text { Mean amplitude of pulse-alone trials } \\
\text { (arbitrary units) }\end{array}$ & $523(117)$ & $579(38.6)$ & $572(36.6)$ & 0.25 & $1 / 97$ & 0.62 & 0.15 & 0.00 \\
\hline $\begin{array}{l}\text { Mean PPI (\%; mean across 3 SOA } \\
\text { conditions: } 30,60,120 \mathrm{~ms})^{c}\end{array}$ & $14.4 \%(4.9)$ & $32.9 \%(1.7)$ & $30.8 \%(1.7)$ & 12.8 & $1 / 93$ & 0.001 & 1.07 & 0.12 \\
\hline $\begin{array}{l}\text { Early habituation of pulse-alone } \\
\text { trials (\%; between first and } \\
\text { second block) }\end{array}$ & $25.9 \%(8.3)$ & $19.6 \%(2.9)$ & $20.4 \%(2.7)$ & 0.59 & $1 / 97$ & 0.45 & 0.24 & 0.01 \\
\hline $\begin{array}{l}\text { Total habituation of pulse-alone } \\
\text { trials (\%; between first and fourth } \\
\text { block) }\end{array}$ & $29.2 \%(11.9)$ & $28.9 \%(3.3)$ & $28.9 \%(3.3)$ & 0.00 & $1 / 97$ & 0.97 & 0.01 & 0.00 \\
\hline $\begin{array}{l}\text { Habituation of pulse-alone trials } \\
\text { across } 4 \text { blocks (linear gradient } \\
\text { coefficient } b \text { ) }\end{array}$ & $-81.3(28.9)$ & $-65.0(8.6)$ & $-67.0(8.3)$ & 0.41 & $1 / 97$ & 0.52 & 0.20 & 0.00 \\
\hline
\end{tabular}

Bold indicates statistical significance.

${ }^{a}$ Effect size Cohen's $d$ ( $d=0.2$ is small, $d=0.5$ is moderate, and $d=0.8$ is a strong effect).

${ }^{b}$ Fisher's exact test.

'ANCOVA, means adjusted by covariates sex and cigarettes per day.

set at $p<0.05$ (two-tailed). Within the Pearson product-moment correlation analyses, the significance level was set at $p<0.01$ (two-tailed) to avoid accumulation of $\alpha$-error. Effect size calculations between two groups refer to Cohen's $d$. For meta-analysis of the genotype effects on PPI across both samples, we used Stouffer's $z$-trend method and Fisher's trend to combine $p$ values (Whitlock, 2005), and the weighted integration method as well as a random-effects model according to Hedges and Olkin to combine effect sizes (Hedges and Olkin, 1985); Armitage's trend test was used to analyze associations between the TCF4 SNP and schizophrenia (Armitage, 1955).

\section{Results}

\section{London sample (healthy volunteers)}

The two genotype groups (homozygous AA-carriers vs C-allele carriers) did not differ regarding age, sex, years of education (YoE), habituation, and startle reactivity (Table 1 ). However, there was not a single smoker in the $\mathrm{C}$-allele group and, therefore, we compared the C-allele group with nonsmoking homozygous AA-carriers in additional analyses (see below).

PPI was strongly affected by TCF4 genotype: carriers of the schizophrenia risk allele $\mathrm{C}$ display decreased PPI levels (Table 1, Fig. $1 A$ ). A $3 \times 2$ (SOA condition $\times$ genotype) repeatedmeasures ANCOVA with sex and CPD as covariates revealed significant main effects for the factors SOA condition $\left(F_{(2,188)}=\right.$ $\left.11.4, p<0.001, \eta_{p^{2}}=0.11\right)$, sex $\left(F_{(1,94)}=7.5, p<0.01, \eta_{p^{2}}=\right.$ $0.07)$, and genotype $\left(F_{(2,94)}=11.2, p<0.001, \eta_{\mathrm{p}^{2}}=0.11\right)$. Moreover, there was a significant interaction of the factors SOA and genotype $\left(F_{(2,188)}=3.0, p<0.05, \eta_{\mathrm{p}^{2}}=0.03\right)$, indicating a differential impact of genotype on the SOA conditions. ANCOVA post hoc test across SOA conditions (corrected for sex and CPD) revealed that the genotype effect was most pronounced in the SOA $120 \mathrm{~ms}$ PPI condition (SOA 30: $F_{(1,94)}=7.4, p<0.01, \eta_{\mathrm{p}^{2}}=$ 0.07; SOA 60: $F_{(1,94)}=4.1, p<0.05, \eta_{\mathrm{p}^{2}}=0.04 ;$ SOA 120: $F_{(1,94)}=$ $\left.14.5, p<0.001, \eta_{\mathrm{p}^{2}}=0.14\right)$. The main effect of SOA reflects the well known nature of PPI to increase with rising SOA from $30 \mathrm{~ms}$ through $120 \mathrm{~ms}$ (Blumenthal, 1999). The effect of sex reflects the known fact that women have generally lower PPI levels than men (pooled SOA conditions: $F_{(1,103)}=10.8, p<0.001, \eta_{p^{2}}=0.10$ ) (Swerdlow et al., 1997).

To exclude a potential impact of smoking on our results, we repeated the PPI analyses after exclusion of smokers (12 CC/AC carriers vs 63 AA carriers). However, PPI was still strongly affected by TCF4 genotype. A $3 \times 2$ (SOA condition $\times$ genotype) repeatedmeasures ANCOVA with sex as a covariate still revealed significant main effects for the factors SOA condition $\left(F_{(2,144)}=7.3, p<0.001\right.$, $\left.\eta_{\mathrm{p}^{2}}=0.09\right)$, sex $\left(F_{(1,72)}=6.4, p<0.05, \eta_{\mathrm{p}^{2}}=0.08\right)$, and genotype $\left(F_{(1,72)}=8.8, p<0.01, \eta_{\mathrm{p}^{2}}=0.11\right)$. The significant SOA $\times$ genotype also remained true $\left(F_{(2,144)}=3.4, p<0.05, \eta_{\mathrm{p}^{2}}=0.05\right)$. The ANCOVA post hoc tests across SOA conditions showed the same effects (SOA 30: $F_{(1,72)}=6.7, p<0.05, \eta_{\mathrm{p}^{2}}=0.09 ; \mathrm{SOA} 60: F_{(1,72)}=$ $3.1, p=0.08, \eta_{\mathrm{p}^{2}}=0.04$; SOA 120: $F_{(1,72)}=11.8, p<0.001, \eta_{\mathrm{p}^{2}}=$ $0.14)$. Introduction of startle amplitude as a covariate also did not change our results. Age, smoking parameters, and YoE did not significantly correlate with any of the psychophysiological parameters.

\section{Bonn sample (schizophrenia patients and high-risk individuals)}

The genotype groups did not differ regarding age, sex, smoking status, medication, and number of high-risk individuals (Table $2)$. There was a moderate effect of genotype on $\operatorname{YoE}(d=0.48)$, suggesting a possible effect of TCF4 genotype on intellectual functioning at least in schizophrenia patients and subjects at risk for schizophrenia (Table 2).

The association between PPI and TCF4 genotype was confirmed in the Bonn sample. Again, carriers of the schizophrenia 

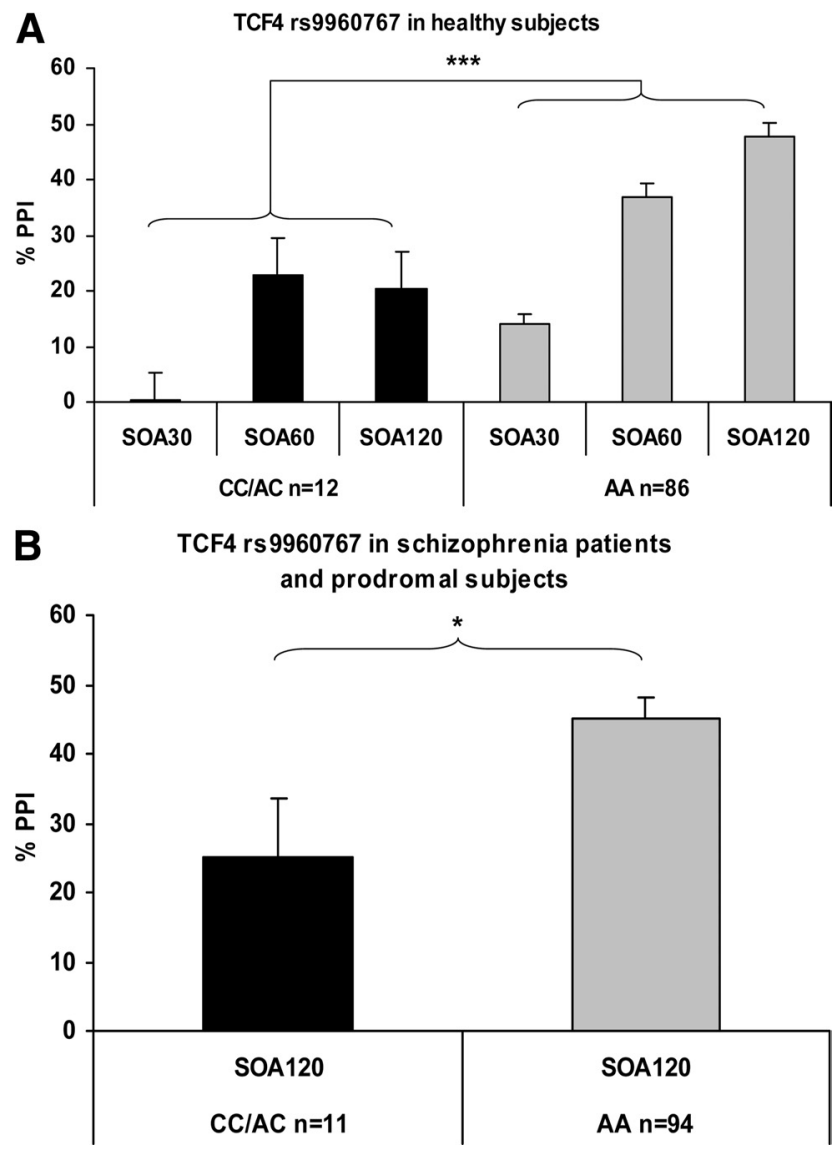

Figure 1. The effects of TCF4 rs9960767 genotype on percentage PPI of the acoustic startle response (means and SEM, adjusted for sex and smoking) in 98 British healthy human volunteers (ANCOVA corrected for sex and smoking: ${ }^{* * *} p<0.001 ; A$ ) and in a combined sample of 68 schizophrenia patients and 37 individuals at high risk for schizophrenia from Germany (ANCOVA corrected for sex, smoking, and antipsychotic medication: $\left.{ }^{*} p<0.05 ; \boldsymbol{B}\right)$. SOA30, S0A60, and SOA120, Stimulus onset asynchrony of 30,60 , and $120 \mathrm{~ms}$, respectively, between prepulse and pulse alone.

risk allele $\mathrm{C}$ revealed lower PPI levels (Table 2, Fig. $1 \mathrm{~B}$ ). An ANCOVA with sex, smoking status, and antipsychotic medication status as covariates revealed a significant main effect of genotype on PPI $\left(F_{(1,100)}=5.1, p<0.05, \eta_{\mathrm{p}^{2}}=0.05\right)$. The influence of the covariate smoking status was not significant $\left(F_{(1,100)}=3.1\right.$, $\left.p=0.08, \eta_{\mathrm{p}^{2}}=0.03\right)$. Introduction of chlorpromazine equivalents as a covariate instead of medication status revealed a similar genotype effect $\left(F_{(1,100)}=4.6, p<0.05, \eta_{\mathrm{p}^{2}}=0.05\right)$. Given that especially atypical antipsychotics could normalize PPI (e.g., Kumari et al., 2000), we also excluded patients treated with atypical antipsychotics from analysis. Despite the limited power of the remaining sample (AA: $n=55$, CC + AC: $n=6$ ), the genotype effect was still significant, while the effect size was improved $\left(F_{(1,57)}=4.11, p<0.05, \eta_{p^{2}}=0.07\right)$. Thus, medication effects could not explain our findings. Moreover, introduction of startle amplitude as a further covariate also did not change our results.

When the factor patient group (high-risk individuals vs schizophrenia patients) was introduced as a further fixed factor into the ANCOVA, the effect of genotype remained significant $\left(F_{(1,99)}=4.3, p<0.05, \eta_{\mathrm{p}^{2}}=0.04\right)$, while neither the factor patient group $\left(F_{(1,99)}=0.74, p=0.39, \eta_{p^{2}}=0.01\right)$ nor the genotype $\times$ patient group interaction $\left(F_{(1,99)}=0.15, p=0.70\right.$, $\left.\eta_{\mathrm{p}^{2}}=0.00\right)$ were significant. Clinical data were not affected by TCF4 genotype. PPI did not correlate with any clinical or demographic data.
Due to the methodological differences, the Bonn schizophrenia spectrum sample and the London controls could not be directly compared regarding startle parameters and PPI. However, in line with many previous studies, the total group of schizophrenia patients and high-risk individuals revealed significantly decreased PPI levels when compared with an equivalent group of healthy controls $\left[t_{(131)}=2.8, p<0.006\right.$, controls: $58.1 \%$ PPI (SEM 3.3), $n=28$ ], who were assessed in Bonn with the same PPI paradigm in a previous study (Quednow et al., 2008a).

\section{Meta-analysis of TCF4 genotype effect on PPI}

To assess the total effect of TCF4 genotype on PPI across both samples, we applied several meta-analytic approaches (Table 3 ). The significance level ranged between 0.0002 and 0.00002 depending on the method used. In general, meta-analytical methods combining effect sizes generated lower $p$ values than methods combining $p$ values. The mean effect size of the genotype effect on PPI was $d=0.90$.

Association between TCF4 genotype, PPI, and schizophrenia To assess the association between the reduced PPI levels and TCF4 rs9960767 genotype, classical odds ratios were calculated (Bland and Altman, 2000). If a clinical criterion of 1.0 SD is applied to define a low PPI level, reduced PPI shows a significant association with the schizophrenia risk allele of the TCF4 SNP within both samples (Table 4). If a more conservative criterion of 1.5 SD is applied as a criterion for diminished PPI, previously significant associations within the London sample and the combined sample still showed marked statistical trends regarding an association. The sex distribution in the $<1.0$ and $<1.5$ SD subgroups was not statistically different from the total sample (across both samples: no criterion: $44.3 \%, 1.0$ SD: $48.3 \%$, 1.5 SD: $43.9 \%$ females). Thus, this effect could not be attributed to an accumulation of females in the low PPI subgroups.

There was no significant association of TCF4 rs9960767 genotype with a diagnosis of schizophrenia (Armitage trend test for the TCF4 C-allele: common odds ratio $=0.71, \chi^{2}=0.92 ; p=$ $0.34)$. However, given that the sample size was rather small and possible effects of population stratification, this result should be interpreted with caution.

\section{Discussion}

The present study is the first to investigate the influence of the schizophrenia risk gene TCF4 rs9960767 on an established endophenotype of schizophrenia. In two independent samples, we could convincingly demonstrate that the schizophrenia risk allele C of the TCF4 rs9960767 SNP is strongly associated with reduced sensorimotor gating. In a meta-analysis of the largest genomewide association study to date, consisting of 12,945 schizophrenia cases and 34,591 controls, this TCF4 SNP was among the most statistically significant findings (Stefansson et al., 2009). The possible role of TCF4 genes in the development of schizophrenia was lately confirmed in a large sample of Han Chinese, in which a neighboring SNP was shown to have an impact on schizophrenia risk (Li et al., 2010). In accordance with recent animal data, showing that transgenic mice overexpressing the TCF4 gene in the brain display decreased sensorimotor gating (Brzózka et al., 2010), this finding suggests that TCF4 plays an important role in the development of early information deficits in schizophrenia at least in a subgroup of patients who display diminished PPI.

The role of TCF4 (also known as E2-2, SEF-2, ME-2, or ITF-2) in the brain and the functional activity of the investigated TCF4 variant on the level of gene expression are not fully understood 
Table 2. Demographic data and psychophysiological parameters of a combined sample of 68 schizophrenia patients and 37 individuals at high risk for schizophrenia from Bonn, Germany, grouped according to their TCF4 rs9960767 genotype (means and SEMs in parentheses, sex and smoking status in frequency data)

\begin{tabular}{|c|c|c|c|c|c|c|c|c|}
\hline TCF4 rs9960767 genotype & $C C+A C$ & $\mathrm{AA}$ & Total & $F$ & $\mathrm{df} / \mathrm{df}_{\mathrm{err}}$ & $p$ & $d^{a}$ & $\eta_{p^{2}}$ \\
\hline$n$ & $11(10.5 \%)$ & $94(89.5 \%)$ & $105(100 \%)$ & & & & & \\
\hline Age & $34.0(4.1)$ & $31.6(1.0)$ & $31.9(1.0)$ & 0.55 & $1 / 104$ & 0.46 & 0.24 & 0.01 \\
\hline Years of education & $13.4(0.7)$ & $14.8(0.3)$ & $14.6(0.3)$ & 2.28 & $1 / 104$ & 0.13 & 0.48 & 0.02 \\
\hline $\operatorname{Men}(\%)^{b}$ & $45.5 \%$ & $63.8 \%$ & $61.9 \%$ & - & 1 & 0.33 & - & - \\
\hline Current smokers (\%) ${ }^{b}$ & $63.6 \%$ & $45.7 \%$ & $47.6 \%$ & - & 1 & 0.34 & - & - \\
\hline High-risk subjects (\%) ${ }^{b}$ & $45.5 \%$ & $34.0 \%$ & $35.2 \%$ & - & 1 & 0.51 & - & - \\
\hline $\begin{array}{l}\text { Medication status (\%; never-medicated/ } \\
\text { typical/atypical antipsychotic) }\end{array}$ & $45.5 / 9.1 / 45.5 \%$ & $47.9 / 10.6 / 41.5 \%$ & $47.6 / 10.5 / 41.9 \%$ & - & 2 & 0.97 & - & - \\
\hline Daily chlorpromazine equivalents ${ }^{c}$ & $205(64.0)$ & $166(23.0)$ & $171(21.6)$ & 0.32 & $1 / 104$ & 0.57 & 0.18 & 0.00 \\
\hline PANSS Positive & $16.3(2.3)$ & $15.5(0.9)$ & $15.6(0.8)$ & 0.09 & $1 / 104$ & 0.76 & 0.10 & 0.00 \\
\hline PANSS Negative & $18.8(3.0)$ & $17.9(0.9)$ & $17.9(0.8)$ & 0.11 & $1 / 104$ & 0.75 & 0.11 & 0.00 \\
\hline PANSS General & $36.8(4.8)$ & $36.8(1.5)$ & $36.8(1.4)$ & 0.00 & $1 / 104$ & 1.00 & 0.00 & 0.00 \\
\hline PANSS Total & $71.9(9.6)$ & $70.6(2.9)$ & 70.7 (2.8) & 0.02 & $1 / 104$ & 0.89 & 0.05 & 0.00 \\
\hline $\begin{array}{l}\text { First block, amplitude of pulse-alone trials } \\
\text { (arbitrary units) }\end{array}$ & $384(74.9)$ & $306(21.0)$ & $314(20.3)$ & 1.38 & $1 / 104$ & 0.24 & 0.37 & 0.01 \\
\hline $\begin{array}{l}\text { Mean amplitude of pulse-alone trials } \\
\text { (arbitrary units) }\end{array}$ & $291(64.1)$ & $232(18.7)$ & $238(18.0)$ & 1.00 & $1 / 104$ & 0.32 & 0.32 & 0.01 \\
\hline Mean percentage PPI (SOA $120 \mathrm{~ms})^{d}$ & $25.0 \%(8.4)$ & $45.2 \%(2.9)$ & $42.6 \%(2.7)$ & 5.13 & $1 / 100$ & 0.03 & 0.73 & 0.05 \\
\hline $\begin{array}{l}\text { Percentage early habituation of pulse-alone } \\
\text { trials (between first and second block) }\end{array}$ & $24.8 \%(4.4)$ & $22.3 \%(3.1)$ & $22.6 \%(2.8)$ & 0.07 & $1 / 104$ & 0.79 & 0.09 & 0.00 \\
\hline $\begin{array}{l}\text { Percentage total habituation of pulse-alone } \\
\text { trials (between first and sixth block) }\end{array}$ & $32.9 \%(7.6)$ & $42.1 \%(3.0)$ & $41.1 \%(2.8)$ & 1.06 & $1 / 104$ & 0.31 & 0.33 & 0.01 \\
\hline $\begin{array}{l}\text { Habituation of pulse-alone trials across } 6 \\
\text { blocks (linear gradient coefficient } b \text { ) }\end{array}$ & $-19.1(5.1)$ & $-20.1(1.9)$ & $-20.0(1.8)$ & 0.03 & $1 / 104$ & 0.86 & 0.05 & 0.00 \\
\hline
\end{tabular}

Bold indicates statistical significance.

${ }^{a}$ Effect size Cohen's $d$ ( $d=0.2$ is small, $d=0.5$ is moderate, and $d=0.8$ is a strong effect).

${ }^{b}$ Fisher's exact test.

Unmedicated patients and high-risk individuals received the value 0 .

${ }^{d}$ ANCOVA, means adjusted by covariates sex, medication (medicated/unmedicated), and smoking status (yes/no).

Table 3. Different meta-analytical approaches to assess TCF4 rs9960767 genotype effects on mean percentage PPI across both investigated samples [healthy volunteers from London, UK $(n=96)$, and a schizophrenia spectrum sample from Bonn, Germany $(n=105)$ ]

\begin{tabular}{lccc}
\hline & Effect size & $Z$ & $p$ value \\
\hline Fisher's trend & - & - & 0.0002 \\
Stouffer's $z$ trend method & - & - & 0.0001 \\
Weighted integration method $^{a}$ & $d+=0.94$ & 4.12 & 0.00002 \\
Random-effects model $^{a}$ & Delta $=0.94$ & 4.12 & 0.00002 \\
\hline
\end{tabular}

${ }^{a}$ According to Hedges and Olkin (1985).

yet, but bHLH transcription factors such as TCF4 seem to play an important role in the development of the mammalian cortex as neuronal progenitors (Ross et al., 2003; Flora et al., 2007). TCF4 belongs to a subfamily of $b H L H$ transcriptional regulators that recognizes the Ephrussi-box ("E-box") binding site on the DNA that usually lies upstream of a gene in a promoter region (Zweier et al., 2007). At early developmental stages, E-box transcription factors such as TCFE2a, TCF12, and TCF4 show wide expression throughout the brain, but only TCF4 displays sustained expression in the adult brain of mice, which is most prominent in the cerebellum, hippocampus, and cortex (Soosaar et al., 1994; Uittenbogaard and Chiaramello, 2000; Brzózka et al., 2010).

TCF4-null knock-out mice die in the first $24 \mathrm{~h}$ after birth (Zhuang et al., 1996; Flora et al., 2007), and haploinsufficiency of the TCF4 gene in humans causes the Pitt-Hopkins syndrome (Zweier et al., 2007; de Pontual et al., 2009)—a neurodevelopmental disease characterized by severe mental retardation, microcephaly, epilepsy, facial dysmorphisms, and intermittent hyperventilation, suggesting that TCF4 is critical for the development of the mammalian nervous system. It was recently shown in
TCF4 knock-out mice that TCF4 plays a unique role especially in the development of the pontine nuclei (Flora et al., 2007). These nuclei are highly interconnected with the pedunculopontine and laterodorsal tegmental nuclei, the caudal pontine reticular nucleus, and the superior colliculus (Vertes et al., 1986; Redgrave et al., 1987; Woolf and Butcher, 1989), which are critical core regions within the CSPP circuitry processing PPI of ASR (Koch, 1999; Fendt et al., 2001; Swerdlow et al., 2001). Although the influence of pontine nuclei on PPI has not been studied so far, one might speculate that developmental changes in these nuclei caused by TCF4 mutations are possibly associated with functional alterations of connections to neighboring brainstem nuclei as well as of the cortico-ponto-cerebellar integration of sensorimotor information. This assumption is also supported by the fact that, in the London sample, PPI was strongly affected by TCF4 genotype across the entire range of SOA conditions-from the "preconscious" $30 \mathrm{~ms}$ SOA to the "conscious" 120 SOA. This pattern suggests that TCF4 genotype probably influences PPI at an early level of information processing. The role of TCF4 in sensorimotor gating could be further investigated by studying PPI in patients with Pitt-Hopkins syndrome (Zweier et al., 2007). However, this mutation is rare and the recruitment of a sufficient sample size as well as the matching would be a demanding exercise.

In the meantime, numerous association studies in healthy humans and schizophrenia patients suggest that PPI is influenced by several genetic variations. It was shown that SNPs of the serotonin-2A receptor $\left(5-H_{2 A} R\right)$, the catechol-Omethyltransferase $(C O M T)$, the dopamine $\mathrm{D}_{3}$ receptor, and the $\alpha 3$ subunit of nicotinic acetylcholine receptor (CHRNA3) may all reliably affect PPI (Quednow et al., 2008b, 2009, 2010; Roussos et 
Table 4. Association of low PPI with the presence of the schizophrenia risk allele C of the TCF4 rs9960767 polymorphism in both investigated samples

\begin{tabular}{|c|c|c|c|c|c|c|c|c|}
\hline Criterion for low PPI levels $^{a}$ & Samples & Total $n$ & $\begin{array}{l}\text { PPI } \\
\text { deficit }(n)\end{array}$ & $\begin{array}{l}\text { TCF4 } \\
\text { C-allele }(n)\end{array}$ & $\begin{array}{l}\text { PPI deficit + } \\
\text { TCF4 C-allele ( } n \text { ) }\end{array}$ & $\begin{array}{l}\text { Odds } \\
\text { ratio }\end{array}$ & $95 \% \mathrm{Cl}$ & $p^{b}$ \\
\hline \multirow[t]{3}{*}{ 1SD below normal } & London healthy controls & 98 & 17 & 12 & 6 & 6.82 & $1.86-24.93$ & 0.006 \\
\hline & Bonn schizophrenia patients + high-risk individuals & 105 & 41 & 11 & 8 & 4.93 & $1.22-19.85$ & 0.022 \\
\hline & London + Bonn & 203 & 58 & 23 & 14 & 4.81 & $1.95-11.87$ & 0.0009 \\
\hline \multirow[t]{3}{*}{$1.5 \mathrm{SD}$ below normal } & London healthy controls & 98 & 9 & 12 & 3 & 4.44 & $0.95-20.89$ & 0.078 \\
\hline & Bonn schizophrenia patients + high-risk individuals & 105 & 32 & 11 & 5 & 2.07 & $0.58-7.35$ & 0.304 \\
\hline & London + Bonn & 203 & 41 & 23 & 8 & 2.38 & $0.93-6.07$ & 0.093 \\
\hline
\end{tabular}

Bold indicates statistical significance. Cl, Confidence interval.

${ }^{a}$ The healthy London sample refers to itself (mean PPI across $30 \mathrm{~ms}, 60 \mathrm{~ms}$, and $120 \mathrm{~ms}$ SOA conditions: $30.8 \pm 17.2 \%$ SD), whereas the schizophrenia spectrum Bonn sample refers to a normal population showing a mean PPI of $58.1 \%$ ( \pm 21.7 SD) at an SOA of $120 \mathrm{~ms}$ measured with the same setup (Quednow et al., 2008a).

${ }^{b}$ Fisher's exact test.

al., 2008a,b; Petrovsky et al., 2010), which parallels well the neural circuitry that underlies and supports PPI of the ASR (Swerdlow and Koob, 1987; Koch, 1999; Fendt et al., 2001; Swerdlow et al., 2001). Thus, it is most likely that each of these genetic polymorphisms contributes a small fraction to the variant expression of PPI in the population. If PPI is an appropriate endophenotype of schizophrenia, as has been proposed, a combination of these SNPs might also contribute to the risk for schizophrenia. Nevertheless, it is not known at present whether and how these SNPs may interact with regard to sensorimotor gating, as the sample sizes investigated so far ( $<250$ subjects) were not suitable to investigate the interactive and epistatic effects even of two SNPs with moderate minor allele frequencies. Further studies with larger sample sizes $(>500)$ are needed to reliably examine these effects. However, since very few schizophrenia patients seem to share identical genomic causation (Need et al., 2009), reduced PPI most likely also arises from several independent genetic variations.

In our samples, schizophrenia was not associated with TCF4 genotype, as was previously shown (Stefansson et al., 2009). However, our sample sizes were too small and had power too low to detect common, relatively low-risk variants such as TCF4, which shows a mean odds ratio of 1.23 (confidence interval: 1.151.32) in a large meta-analysis (Stefansson et al., 2009). Moreover, the two samples were recruited in different European countries, limiting the direct comparability because of possible population stratification effects, which were not statistically controlled by a standard panel of ancestry-informative SNPs. However, the investigation of the association of TCF4 and schizophrenia was not the focus of the present study. Interestingly, low PPI levels have shown stronger associations with the TCF4 risk allele $\mathrm{C}$ than schizophrenia per se: depending on the criterion for decreased PPI ( 1 or 1.5 SD below normal), odds ratios of 2.38 and 4.81 were calculated for the entire sample (Table 4 ). When considering the effect size measures according to Cohen (1988), a similar pattern arises: In the meta-analysis, the association of a diagnosis of schizophrenia with TCF4 genotype displayed only a very small effect size of $w=0.09$ (Stefansson et al., 2009). In contrast, the association of the schizophrenia endophenotype PPI with TCF4 showed a strong effect size of $d=0.90$ averaged across both samples. Thus, the endophenotype approach could boost the power of a genetic study compared with the approach of classical categorization of the mental illness using the current diagnostic manuals. Furthermore, given that TCF4 genotype was significantly associated with PPI reduction, a combination of TCF4 genotype status and low PPI levels might be a promising marker for the early detection of schizophrenia.

The study has some limitations. (I) The total size of our samples seems to be modest. However, compared to other studies in the field, our study with a sample size of $n=203$ still belongs to the best-powered PPI genetics studies. In contrast to many other association studies, we recruited a highly homogeneous sample regarding ethnicity because we only included Caucasians from Middle Europe with self-reported grandparents of Middle European origin. Thus, we have a modest but clear and well selected sample, which might be preferable compared to ethnically mixed but larger samples.

(II) Are the sample sizes of subjects carrying the C-allele too low for a reliable estimation of the group PPI levels? We do not assume this for three reasons: (1) We tested two independent samples as we aimed to obtain an internal replication of our results especially to avoid spurious results. The replication of the initial results in an independent sample makes it statistically highly unlikely that both results could be accidental or spurious. (2) To estimate the strength of the results, we conducted several meta-analyses with different approaches (Table 3). They all revealed a very strong genotype effect of the investigated TCF4 SNP on PPI across both samples ( $p<0.0001-0.00002$, mean Cohen's $d=0.90)$, which is the strongest genotype effect on PPI that has been shown across more than one tested sample so far. (3) In total, we have 23 subjects carrying the $\mathrm{C}$-allele, which is sufficient for a reliable estimation of the mean PPI of a group. Of these 23 subjects, 14 (61\%) displayed decreased PPI levels, when compared to the total sample (Table 4), which is again an expression of the strong genotype effect of TCF4 on PPI. Moreover, most of the positive results of previous genetic PPI studies are based on comparable or smaller SNP subgroups (e.g., Hong et al., 2008; Quednow et al., 2008b, 2009, 2010; Roussos et al., 2008a,b). Thus, compared to the field of PPI genetics (as well as compared to many clinical PPI studies), a total of 23 risk allele carriers should be sufficient for a reliable and valid estimation of the mean PPI levels.

(III) The two independent samples were assessed with slightly different PPI paradigms, which preclude the direct comparison of the samples regarding PPI. Therefore, we decided to analyze the genotype effects only within each sample and to subsequently pool the $p$ values and effect sizes of the genotype effects in metaanalyses to overcome these methodological differences. The replication of the genotype effects within each of the samples and across study sites despite some procedural differences underscores the robustness of our findings.

(IV) All participants were screened for drug use within the SCID-I interview, but we only obtained drug urine toxicologies from our schizophrenia patients to rule out current drug use. Thus, we could exclude acute illicit drug effects on PPI only in a subset of the total subjects. However, given the strict inclusion criteria, it is highly unlikely that a significant number of subjects would have been taking drugs, and even less likely that a gene- 
phenotype association that is observed in two independent samples could be accounted for by this.

(V) Finally, estimations of the genetic effect based on novel association findings tend to be upwardly biased due to a phenomenon also known as the "winner's curse" (Xiao and Boehnke, 2009). However, given that we replicated our finding in a second sample and considering the fact that the effect size of TCF4 genotype effect seems to be one of the strongest genotype effects on PPI reported so far (e.g., Quednow et al., 2008b, 2009, 2010; Roussos et al., 2008a,b, 2009; Petrovsky et al., 2010), it is highly likely that this effect will survive further investigation.

In conclusion, our results suggest that TCF4 variants could play an important role in the development of diminished sensorimotor gating in schizophrenia. These deficits might arise from developmental changes of brainstem nuclei. As subjects carrying the TCF4 rs9960767 C-allele robustly showed diminished PPI levels, an extended endophenotype including PPI and TCF4 genotype status warrants testing as an early indicator for a developing psychosis (Prasad and Keshavan, 2008).

\section{References}

Anokhin AP, Heath AC, Myers E, Ralano A, Wood S (2003) Genetic influences on prepulse inhibition of startle reflex in humans. Neurosci Lett 353:45-48.

Armitage P (1955) Tests for linear trends in proportions and frequencies. Biometrics 11:375-386.

Bland JM, Altman DG (2000) Statistics notes. The odds ratio. BMJ 320:1468.

Blumenthal TD (1999) Short lead interval startle modification. In: Startle modification. Implications for neuroscience, cognitive sciences, and clinical science (Dawson ME, Schell AM, Böhmelt AH, eds), pp 51-71. Cambridge, UK: Cambridge UP.

Braff DL, Grillon C, Geyer MA (1992) Gating and habituation of the startle reflex in schizophrenic patients. Arch Gen Psychiatry 49:206-215.

Braff DL, Geyer MA, Swerdlow NR (2001) Human studies of prepulse inhibition of startle: normal subjects, patient groups, and pharmacological studies. Psychopharmacology (Berl) 156:234-258.

Brzózka MM, Radyushkin K, Wichert SP, Ehrenreich H, Rossner MJ (2010) Cognitive and sensorimotor gating impairments in transgenic mice overexpressing the schizophrenia susceptibility gene Tcf4 in the brain. Biol Psychiatry 68:33-40.

Cadenhead KS, Geyer MA, Braff DL (1993) Impaired startle prepulse inhibition and habituation in patients with schizotypal personality disorder. Am J Psychiatry 150:1862-1867.

Cadenhead KS, Swerdlow NR, Shafer KM, Diaz M, Braff DL (2000) Modulation of the startle response and startle laterality in relatives of schizophrenic patients and in subjects with schizotypal personality disorder: evidence of inhibitory deficits. Am J Psychiatry 157:1660-1668.

Cohen J (1988) Statistical power analysis for the behavioral sciences, Ed 2. Hillsdale, NJ: Lawrence Erlbaum.

de Pontual L, Mathieu Y, Golzio C, Rio M, Malan V, Boddaert N, Soufflet C, Picard C, Durandy A, Dobbie A, Heron D, Isidor B, Motte J, NewburryEcob R, Pasquier L, Tardieu M, Viot G, Jaubert F, Munnich A, Colleaux L, et al. (2009) Mutational, functional, and expression studies of the TCF4 gene in Pitt-Hopkins syndrome. Hum Mutat 30:669-676.

Fendt M, Li L, Yeomans JS (2001) Brain stem circuits mediating prepulse inhibition of the startle reflex. Psychopharmacology (Berl) 156:216-224.

Flora A, Garcia JJ, Thaller C, Zoghbi HY (2007) The E-protein Tcf4 interacts with Math1 to regulate differentiation of a specific subset of neuronal progenitors. Proc Natl Acad Sci U S A 104:15382-15387.

Frommann I, Pukrop R, Brinkmeyer J, Bechdolf A, Ruhrmann S, Berning J, Decker P, Riedel M, Möller HJ, Wölwer W, Gaebel W, Klosterkötter J, Maier W, Wagner M (2010) Neuropsychological profiles in different at-risk states of psychosis: executive control impairment in the earlyand additional memory dysfunction in the late-prodromal state. Schizophr Bull. Advance online publication. doi:10.1093/schbul/sbp155.

Gottesman II, Gould TD (2003) The endophenotype concept in psychiatry: etymology and strategic intentions. Am J Psychiatry 160:636-645.

Graham FK (1975) The more or less startling effects of weak prestimulation. Psychophysiology 12:238-248.
Greenwood TA, Braff DL, Light GA, Cadenhead KS, Calkins ME, Dobie DJ, Freedman R, Green MF, Gur RE, Gur RC, Mintz J, Nuechterlein KH, Olincy A, Radant AD, Seidman LJ, Siever LJ, Silverman JM, Stone WS, Swerdlow NR, Tsuang DW, et al. (2007) Initial heritability analyses of endophenotypic measures for schizophrenia: the consortium on the genetics of schizophrenia. Arch Gen Psychiatry 64:1242-1250.

Häfner H, Maurer K, Ruhrmann S, Bechdolf A, Klosterkötter J, Wagner M, Maier W, Bottlender R, Möller HJ, Gaebel W, Wölwer W (2004) Early detection and secondary prevention of psychosis: facts and visions. Eur Arch Psychiatry Clin Neurosci 254:117-128.

Heatherton TF, Kozlowski LT, Frecker RC, Fagerström KO (1991) The Fagerstrom test for nicotine dependence: a revision of the Fagerstrom tolerance questionnaire. Br J Addict 86:1119-1127.

Hedges LV, Olkin I (1985) Statistical methods for meta-analysis. New York: Academic.

Hong LE, Wonodi I, Lewis J, Thaker GK (2008) Nicotine effect on prepulse inhibition and prepulse facilitation in schizophrenia patients. Neuropsychopharmacology 33:2167-2174.

Kay SR, Opler LA, Fiszbein A (1992) Positive and Negative Syndrome Scale (PANSS). Manual. North Tonawanda, NY: Multi-Health Systems.

Koch M (1999) The neurobiology of startle. Prog Neurobiol 59:107-128.

Kumari V, Gray JA (1999) Smoking withdrawal, nicotine dependence and prepulse inhibition of the acoustic startle reflex. Psychopharmacology (Berl) 141:11-15.

Kumari V, Soni W, Mathew VM, Sharma T (2000) Prepulse inhibition of the startle response in men with schizophrenia: effects of age of onset of illness, symptoms, and medication. Arch Gen Psychiatry 57:609-614.

Kumari V, Das M, Zachariah E, Ettinger U, Sharma T (2005a) Reduced prepulse inhibition in unaffected siblings of schizophrenia patients. Psychophysiology 42:588-594.

Kumari V, Antonova E, Zachariah E, Galea A, Aasen I, Ettinger U, Mitterschiffthaler MT, Sharma T (2005b) Structural brain correlates of prepulse inhibition of the acoustic startle response in healthy humans. Neuroimage 26:1052-1058.

Li T, Li Z, Chen P, Zhao Q, Wang T, Huang K, Li J, Li Y, Liu J, Zeng Z, Feng G, He L, Shi Y (2010) Common variants in major histocompatibility complex region and TCF4 gene are significantly associated with schizophrenia in Han Chinese. Biol Psychiatry 68:671-673.

Ludewig K, Geyer MA, Vollenweider FX (2003) Deficits in prepulse inhibition and habituation in never-medicated, first-episode schizophrenia. Biol Psychiatry 54:121-128.

Need AC, Ge D, Weale ME, Maia J, Feng S, Heinzen EL, Shianna KV, Yoon W, Kasperaviciūte D, Gennarelli M, Strittmatter WJ, Bonvicini C, Rossi G, Jayathilake K, Cola PA, McEvoy JP, Keefe RS, Fisher EM, St Jean PL, Giegling I, et al. (2009) A genome-wide investigation of SNPs and CNVs in schizophrenia. PLoS Genet 5:e1000373.

Parwani A, Duncan EJ, Bartlett E, Madonick SH, Efferen TR, Rajan R, Sanfilipo M, Chappell PB, Chakravorty S, Gonzenbach S, Ko GN, Rotrosen JP (2000) Impaired prepulse inhibition of acoustic startle in schizophrenia. Biol Psychiatry 47:662-669.

Petrovsky N, Quednow BB, Ettinger U, Schmechtig A, Mössner R, Collier DA, Kühn K-U, Maier W, Wagner M, Kumari V (2010) Sensorimotor gating is associated with CHRNA3 polymorphisms in schizophrenia and healthy volunteers. Neuropsychopharmacology 35:1429-1439.

Prasad KM, Keshavan MS (2008) Structural cerebral variations as useful endophenotypes in schizophrenia: do they help construct "extended endophenotypes"? Schizophr Bull 34:774-790.

Quednow BB, Kühn KU, Beckmann K, Westheide J, Maier W, Wagner M (2006a) Attenuation of the prepulse inhibition of the acoustic startle response within and between sessions. Biol Psychol 71:256-263.

Quednow BB, Wagner M, Westheide J, Beckmann K, Bliesener N, Maier W, Kühn KU (2006b) Sensorimotor gating and habituation of the startle response in schizophrenic patients randomly treated with amisulpride or olanzapine. Biol Psychiatry 59:536-545.

Quednow BB, Frommann I, Berning J, Kühn KU, Maier W, Wagner M (2008a) Impaired sensorimotor gating of the acoustic startle response in the prodrome of schizophrenia. Biol Psychiatry 64:766-773.

Quednow BB, Kühn KU, Mössner R, Schwab SG, Schuhmacher A, Maier W, Wagner M (2008b) Sensorimotor gating of schizophrenia patients is influenced by 5-HT2A receptor polymorphisms. Biol Psychiatry 64:434-437.

Quednow BB, Schmechtig A, Ettinger U, Petrovsky N, Collier DA, Vollenweider FX, Wagner M, Kumari V (2009) Sensorimotor gating depends on poly- 
morphisms of the serotonin-2A receptor and catechol-O-methyltransferase, but not on neuregulin-1 Arg38GIn genotype: a replication study. Biol Psychiatry 66:614-620.

Quednow BB, Wagner M, Mössner R, Maier W, Kühn KU (2010) Sensorimotor gating of schizophrenia patients depends on catechol O-methyltransferase Val158Met polymorphism. Schizophr Bull 36:341-346.

Redgrave P, Mitchell IJ, Dean P (1987) Descending projections from the superior colliculus in rat: a study using orthograde transport of wheatgermagglutinin conjugated horseradish peroxidase. Exp Brain Res 68:147-167.

Ross SE, Greenberg ME, Stiles CD (2003) Basic helix-loop-helix factors in cortical development. Neuron 39:13-25.

Roussos P, Giakoumaki SG, Bitsios P (2008a) The dopamine D(3) receptor Ser9Gly polymorphism modulates prepulse inhibition of the acoustic startle reflex. Biol Psychiatry 64:235-240.

Roussos P, Giakoumaki SG, Rogdaki M, Pavlakis S, Frangou S, Bitsios P (2008b) Prepulse inhibition of the startle reflex depends on the catechol O-methyltransferase Val158Met gene polymorphism. Psychol Med 38:1651-1658.

Roussos P, Giakoumaki SG, Bitsios P (2009) A risk PRODH haplotype affects sensorimotor gating, memory, schizotypy, and anxiety in healthy male subjects. Biol Psychiatry 65:1063-1070.

Soosaar A, Chiaramello A, Zuber MX, Neuman T (1994) Expression of basic-helix-loop-helix transcription factor ME2 during brain development and in the regions of neuronal plasticity in the adult brain. Brain Res Mol Brain Res 25:176-180.

Stefansson H, Ophoff RA, Steinberg S, Andreassen OA, Cichon S, Rujescu D, Werge T, Pietiläinen OP, Mors O, Mortensen PB, Sigurdsson E, Gustafsson $\mathrm{O}$, Nyegaard $\mathrm{M}$, Tuulio-Henriksson A, Ingason A, Hansen $\mathrm{T}$, Suvisaari J, Lonnqvist J, Paunio T, Børglum AD, et al. (2009) Common variants conferring risk of schizophrenia. Nature 460:744-747.

Swerdlow NR, Koob GF (1987) Dopamine, schizophrenia, mania, and depression: toward a unified hypothesis of cortico-striatopallido-thalamic function. Behav Brain Sci 10:197-208.
Swerdlow NR, Hartman PL, Auerbach PP (1997) Changes in sensorimotor inhibition across the menstrual cycle: implications for neuropsychiatric disorders. Biol Psychiatry 41:452-460.

Swerdlow NR, Geyer MA, Braff DL (2001) Neural circuit regulation of prepulse inhibition of startle in the rat: current knowledge and future challenges. Psychopharmacology (Berl) 156:194-215.

Swerdlow NR, Light GA, Cadenhead KS, Sprock J, Hsieh MH, Braff DL (2006) Startle gating deficits in a large cohort of patients with schizophrenia: relationship to medications, symptoms, neurocognition, and level of function. Arch Gen Psychiatry 63:1325-1335.

Uittenbogaard M, Chiaramello A (2000) Differential expression patterns of the basic helix-loop-helix transcription factors during aging of the murine brain. Neurosci Lett 280:95-98.

Vertes RP, Martin GF, Waltzer R (1986) An autoradiographic analysis of ascending projections from the medullary reticular formation in the rat. Neuroscience 19:873-898.

Whitlock MC (2005) Combining probability from independent tests: the weighted Z-method is superior to Fisher's approach. J Evol Biol 18:1368-1373.

Woolf NJ, Butcher LL (1989) Cholinergic systems in the rat brain: IV. Descending projections of the pontomesencephalic tegmentum. Brain Res Bull 23:519-540.

Xiao R, Boehnke M (2009) Quantifying and correcting for the winner's curse in genetic association studies. Genet Epidemiol 33:453-462.

Zhuang Y, Cheng P, Weintraub H (1996) B-lymphocyte development is regulated by the combined dosage of three basic helix-loop-helix genes, E2A, E2-2, and HEB. Mol Cell Biol 16:2898-2905.

Zweier C, Peippo MM, Hoyer J, Sousa S, Bottani A, Clayton-Smith J, Reardon W, Saraiva J, Cabral A, Gohring I, Devriendt K, de Ravel T, Bijlsma EK, Hennekam RC, Orrico A, Cohen M, Dreweke A, Reis A, Nurnberg P, Rauch A (2007) Haploinsufficiency of TCF4 causes syndromal mental retardation with intermittent hyperventilation (Pitt-Hopkins syndrome). Am J Hum Genet 80:994-1001. 